\title{
Optical and NIR observations of the afterglow of GRB 020813 ${ }^{\star}$
}

\author{
S. Covino ${ }^{1}$, D. Malesani ${ }^{1}$, F. Tavecchio ${ }^{1}$, L. A. Antonelli ${ }^{2}$, A. Arkharov ${ }^{3}$, A. Di Paola ${ }^{2}$, D. Fugazza ${ }^{4}$, G. Ghisellini ${ }^{1}$, \\ V. Larionov ${ }^{5,6}$, D. Lazzati $^{7}$, F. Mannucci ${ }^{8}$, N. Masetti ${ }^{9}$, R. Barrena ${ }^{4}$, S. Benetti ${ }^{10}$, A. J. Castro-Tirado ${ }^{11}$, \\ S. Di Serego Alighieri ${ }^{12}$, F. Fiore ${ }^{2}$, F. Frontera ${ }^{9,13}$, A. Fruchter ${ }^{14}$, F. Ghinassi ${ }^{4}$, M. Gladders ${ }^{15}$, P. B. Hall ${ }^{16,17}$, \\ G. L. Israel ${ }^{2}$, S. Klose ${ }^{18}$, A. Magazzù ${ }^{4}$, E. Palazzi ${ }^{9}$, M. Pedani ${ }^{4}$, E. Pian ${ }^{19}$, \\ P. Romano ${ }^{1}$, M. Stefanon ${ }^{1}$, and L. Stella ${ }^{2}$
}

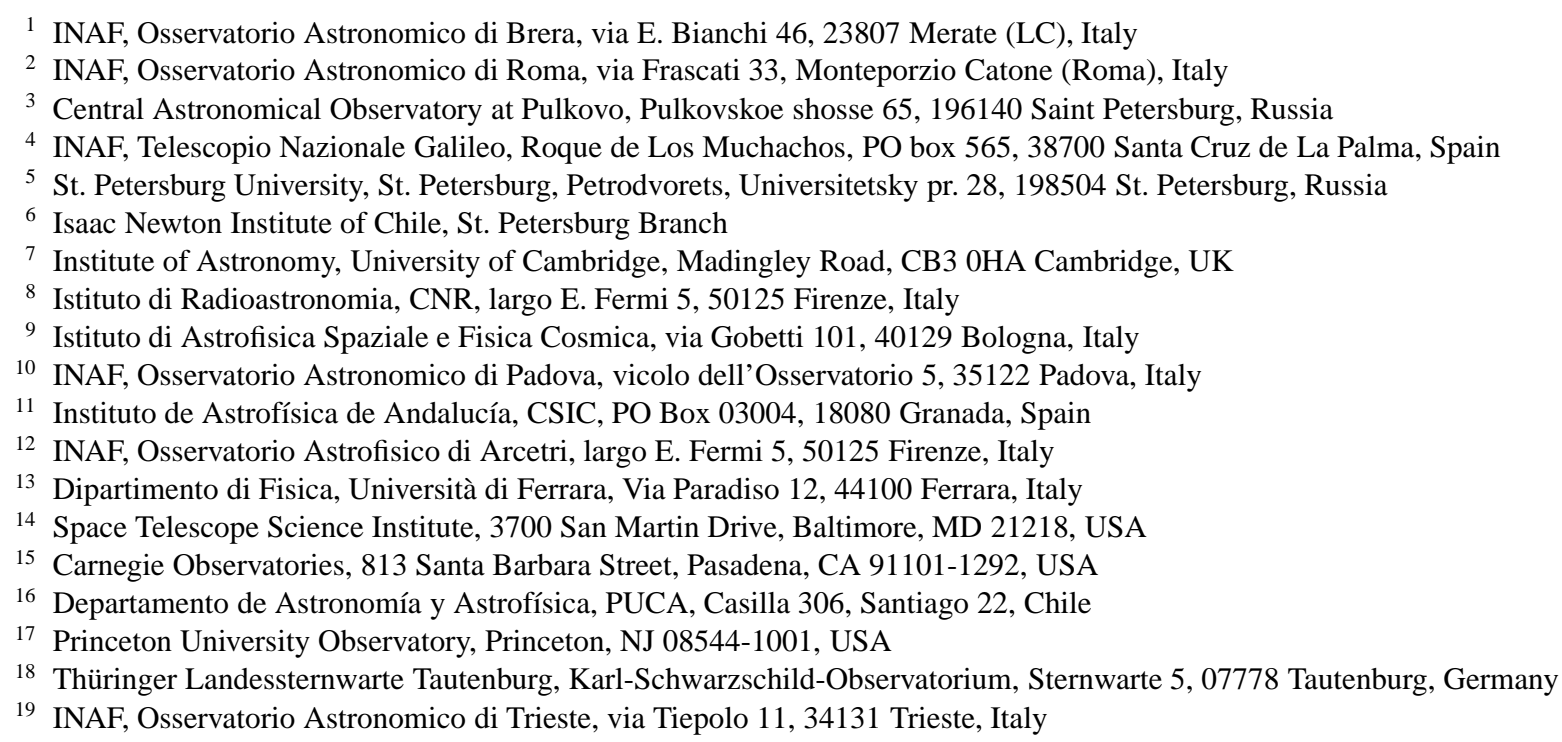

Received 10 October 2002 / Accepted 5 April 2003

\begin{abstract}
We present optical and near-infrared (NIR) photometry of the bright afterglow of GRB 020813. Our data span from 3 hours to 4 days after the GRB event. A rather sharp achromatic break is present in the light curve, 14 hours after the trigger. In the framework of jetted fireballs, this break corresponds to a jet half-opening angle of $1.9^{\circ} \pm 0.2^{\circ}$, the smallest value ever inferred for a GRB. We discuss our results in the framework of currently available models, and find that they have problems in explaining the joint temporal and spectral properties, and in particular the slow decay before the break.
\end{abstract}

Key words. gamma rays: bursts - radiation mechanisms: non-thermal

\section{Introduction}

Since the discovery of the first afterglow of a gamma-ray burst (GRB; Costa et al. 1997; van Paradjis et al. 1997), our knowledge of these mysterious explosions has rapidly increased. The huge energetics implied by their cosmological distance (e.g. Metzger et al. 1997) has severely constrained existing models, leading to the standard internal/external shock scenario (e.g. Piran 1999; Mészáros 2002 and references therein). According

Send offprint requests to: S. Covino, e-mail: covino@mi . astro.it

* Based on observations partly made with ESO telescopes at the Paranal Observatories under programme Id 69.D-0461 and with the Italian TNG telescope under programme TAC 8_01(47). to this model, the prompt gamma-ray emission is produced by internal collisions in a relativistic blastwave, while the afterglow originates by the slowing down of the same fireball in the surrounding medium.

An important question still remains unsettled: are the fireballs spheres or jets? In the latter case, a steepening in the light curve is expected when the bulk Lorentz factor of the fireball equals the inverse of the opening angle of the jet (Rhoads 1999). This steepening must occur at all frequencies and at the same time ("achromatic" break). To date, there are several possible examples of such behaviour in the optical band. The most convincing cases are perhaps GRB 990510 (e.g. Israel et al. 1999; Harrison et al. 1999; Stanek et al. 1999) 


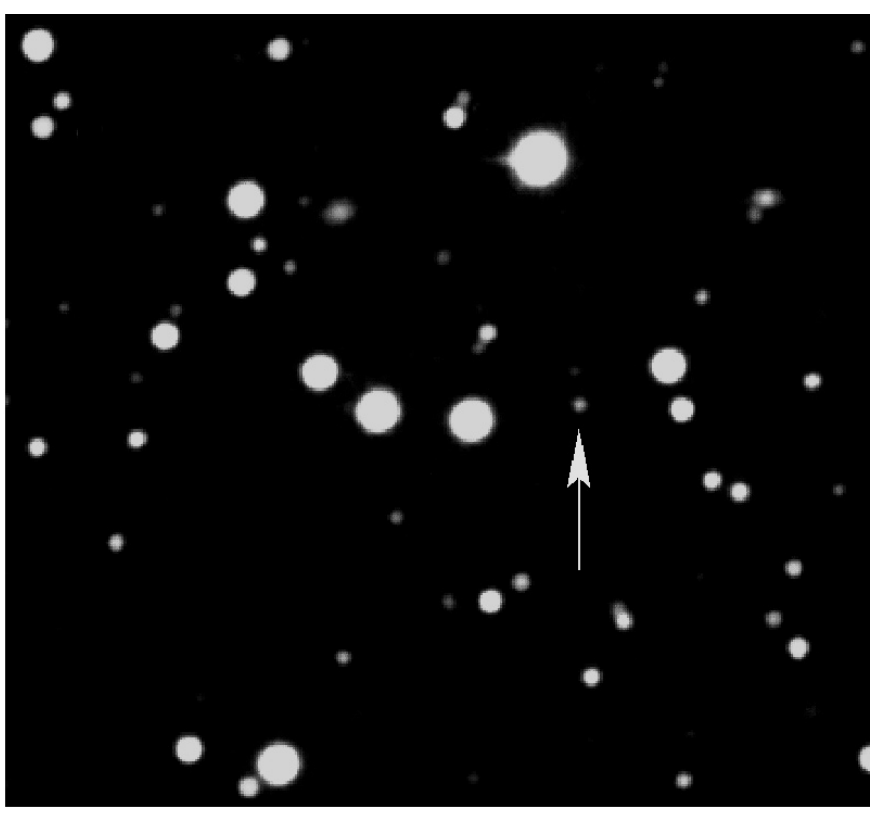

Fig. 1. The field of GRB $020813\left(\sim 1.5^{\prime} \times 1.5^{\prime}\right)$, as imaged by TNGDOLORES on Aug. 15 UT. The afterglow is marked by an arrow. This is a composite image from $B, V$, and $R$ filters. North is up, East is left.

and GRB 010222 (e.g. Masetti et al. 2001; Sagar et al. 2001; Stanek et al. 2001), for which the break times were $\sim 1.5$ days and $\sim 0.7$ days respectively.

GRB 020813 was detected on 2002 August 13 by the HETE-2 spacecraft at 2:44:19 UT (Villasenor et al. 2002). It was a bright event, lasting more than $125 \mathrm{~s}$, with a fluence of $\sim 3.8 \times 10^{-5} \mathrm{erg} \mathrm{cm}^{-2}(25 \div 100 \mathrm{keV})$ as reported by Ulysses (Hurley et al. 2002). The rapid coordinate dissemination and the small HETE-2 error box ( $4^{\prime}$ radius) allowed Fox et al. (2002) and Gladders \& Hall (2002a) to independently identify a bright optical counterpart with position $\alpha_{2000}=19^{\mathrm{h}} 46^{\mathrm{m}} 41^{\mathrm{s}} .9$, $\delta_{2000}=-19^{\circ} 36^{\prime} 04^{\prime \prime} 8$, just 1.9 hours after the trigger. The spectroscopic redshift, determined with KECK 1-LRIS, was $z=1.255$ (Barth et al. 2003). Subsequent observations by various groups allowed a preliminary sampling of the light curve, which exhibited a steepening in the optical bands beginning a few hours after the GRB trigger (e.g. Bloom et al. 2002). Polarimetric observations were carried out by Barth et al. (2003) and Covino et al. (2002), yielding positive polarization signals at the level of $P=(2.9 \pm 0.1) \%$ and $P=(1.17 \pm 0.16) \%$, 4.7 hours and 23 hours after the burst, respectively (both measurements are uncorrected for the small Galactic-induced polarization). The afterglow was also observed in the $\mathrm{X}$-ray band $(0.6 \div 6 \mathrm{keV})$ by Chandra, as a bright fading source with an average flux $F \sim 1.9 \times 10^{-12} \mathrm{erg} \mathrm{cm}^{-2} \mathrm{~s}^{-1}$ (Butler et al. 2003). A positive radio detection was obtained by Frail \& Berger (2002), with $F_{v}=300 \mu \mathrm{Jy}$ at $8.46 \mathrm{GHz}$. Only upper limits were reported in the millimeter region (Bremer \& Castro-Tirado 2002; Bertoldi et al. 2002).

\section{Data collection, reduction, and analysis}

We began observing the optical afterglow of GRB 020813 (Fig. 1) on Aug. 13.99 UT, with the ESO VLT-UT3 (Melipal),

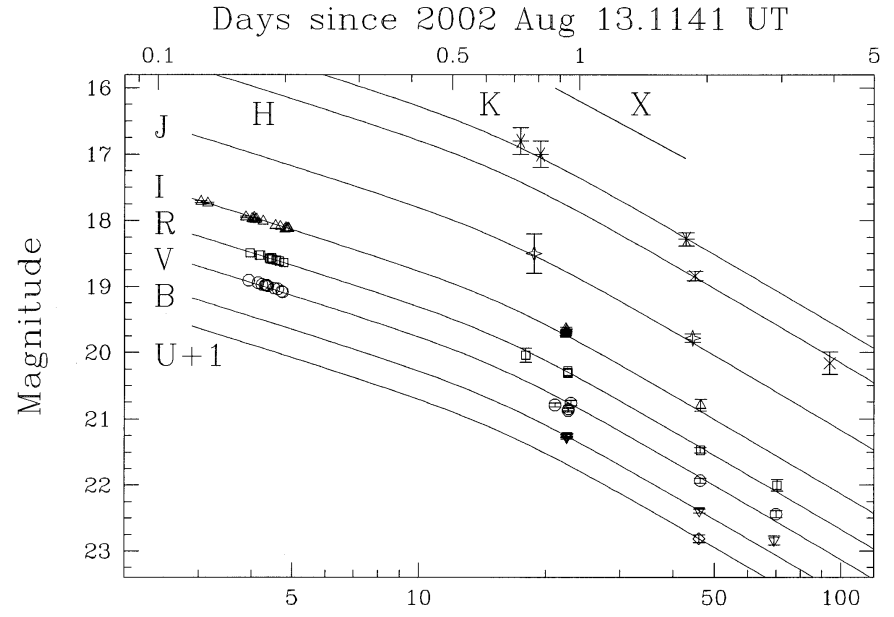

Hours since 2002 Aug 13.1141 UT

Fig. 2. Optical and NIR light curves of GRB 020813 afterglow, with our best fit according to Eq. (1) (dashed lines); refer to Table 1 for fit parameters, and to the web page ${ }^{3}$ for the numerical values. For the first night $(t<5 \mathrm{~h})$, error bars are smaller than symbols and are not plotted. The thick solid line shows the (arbitrarily normalized) X-ray decay.

equipped with FORS 1 and a $V$ filter in imaging polarimetric mode; during the subsequent nights (Aug. 15, 16, and 17), we monitored the optical/NIR light curve using the $3.6 \mathrm{~m}$ Telescopio Nazionale Galileo (TNG) in the Canary Islands. UBVRI and JHK data were acquired with the DOLORES and NICS instruments respectively. Additional NIR images $(J$ and $K$ ) were taken with the AZT24 $1.1 \mathrm{~m}$ telescope in Campo Imperatore (L'Aquila, Italy) on Aug. 13. We then added to our photometric set the publicly avaliable data ${ }^{1}$ of Mike Gladders \& Pat Hall (2002b), acquired with the Baade $6.5 \mathrm{~m}$ (Magellan 1) telescope. Standard stars were imaged during the night of Aug. 16 with the TNG. Data reduction and calibration were carried out following standard procedures, as implemented in the Eclipse package (version 4.2.1; Devillard 1997), while photometry was performed by means of GAIA ${ }^{2}$. All photometric data, shown in Fig. 2, are posted online ${ }^{3}$.

We modelled the afterglow light curve with the functional form first proposed by Beuermann et al. (1999):

$F_{v}(t)=\frac{F_{v}^{*}}{\left[\left(t / t_{\mathrm{b}}\right)^{k \delta_{1}}+\left(t / t_{\mathrm{b}}\right)^{k \delta_{2}}\right]^{1 / k}}$

here $F_{v}$ is the (monochromatic) flux at frequency $v$, and $t$ is the time elapsed from the GRB; this expression reduces to $F_{v} \propto t^{-\delta_{1}}$ for $t \ll t_{\mathrm{b}}$ and $F_{v} \propto t^{-\delta_{2}}$ for $t \gg t_{\mathrm{b}} ; t_{\mathrm{b}}$ is the break time at which the transition between the two regimes occurs, and $k>0$ describes how fast this transition takes place (the larger $k$ the sharper the transition); $F_{v}^{*}$ is a normalization constant. We started by fitting the $V, R$ and $I$ bands, which are sampled closely enough to constrain $t_{\mathrm{b}}$ and $\delta_{1}$. To within the errors, the inferred parameters, summarized in Table 1, were

\footnotetext{
${ }^{1}$ ftp://ftp.ociw.edu/pub/gladders/GRB/GRB020813

2 http://star-www.dur.ac.uk/ pdraper/gaia/gaia.html

3 http://www.merate.mi.astro.it/ malesani/GRB/020813
} 
Table 1. Fitted parameters describing the temporal evolution of the afterglow. $\delta_{1}$ and $\delta_{2}$ are the early- and late-time temporal slopes, and $t_{\mathrm{b}}$ is the break time (measured in hours since Aug. 13.1141 UT); see Eq. (1). Errors are 1- $\sigma$.

\begin{tabular}{llll}
\hline \hline Band & $\delta_{1}$ & $\delta_{2}$ & $t_{\mathrm{b}}(\mathrm{h})$ \\
\hline$V$ & $0.84 \pm 0.11$ & $1.34 \pm 0.06$ & $11.9 \pm 6.4$ \\
$R$ & $0.76 \pm 0.05$ & $1.46 \pm 0.04$ & $13.7 \pm 1.2$ \\
$I$ & $0.80 \pm 0.03$ & $1.43 \pm 0.12$ & $16.7 \pm 1.7$ \\
\hline All & $0.78 \pm 0.02$ & $1.44 \pm 0.04$ & $14.2 \pm 0.8$ \\
\hline
\end{tabular}

determined to be the same between the different bands, even if there is a marginal hint (not statistically significant) for a correlation between $t_{\mathrm{b}}$ and the frequency. We caution that $\delta_{2}$ could also be larger, since our sampling does not extend very much after $t_{\mathrm{b}}$. By fitting simultaneously the whole dataset, including $U, B$ and NIR data, we got our best values. As shown in Fig. 2, at early times the decay was fairly slow, similar to that seen in other bursts with an early break (e.g. GRB 010222: Masetti et al. 2001). After $t_{\mathrm{b}}$, the slope increased by $0.65 \pm 0.05$. In all fits, the parameter $k$, though poorly constrained, was always $\gtrsim 5$, indicating a very sharp transition. This is confirmed by the fact that early data $(t<5 \mathrm{~h})$ are well fitted by a single powerlaw, showing that the steepening had not yet started at this time. This is in contrast with the finding of Gladders \& Hall (2002c), who claim a steepening in the I-band between 3 and 5 hours after the GRB.

To further check if the break is really achromatic, we studied the spectral properties of the afterglow, using our photometric multiband data points, both before and after the break (Fig. 3). We first corrected for Galactic reddening, assuming a neutral hydrogen column density ${ }^{4}$ of $N_{\mathrm{H}}=7.52 \times 10^{20} \mathrm{~cm}^{-2}$, corresponding to a $V$-band absorption $A_{V}=0.42$ (Predehl \& Schmitt 1995). Individual powerlaw fits to each dataset yield the spectral indices reported in Table $2\left(F_{v} \propto v^{-\alpha}\right)$, consistent with $\alpha$ remaining constant during all the observations. This is a confirmation that the break is indeed achromatic. A simultaneous fit to the full dataset (six spectra) gives the best value $\alpha=1.04 \pm 0.03$. The colors are $B-V=0.32 \pm 0.05$, $V-R=0.39 \pm 0.06, R-I=0.45 \pm 0.10$, within the range of the sample by Šimon et al. (2001). The chi-square of the fit is $\chi^{2}=82$ for 17 degrees of freedom. Such large value is due to the use of data coming from different telescopes.

\section{Discussion}

The afterglow of GRB 020813 represents a new case in which the optical light curve shows an achromatic break. The break was quite sharp and occurred early, similar to that of GRB 010222. The slope after the break $(\sim 1.4)$ was typical of afterglows at times between 1 and 10 days after the GRB. Since observations are often performed during this range of days (and only rarely earlier), it is possible that many such breaks have been missed in the past. An intriguing example of this

\footnotetext{
${ }^{4}$ http://heasarc.gsfc.nasa.gov/docs/corp/tools.html
}

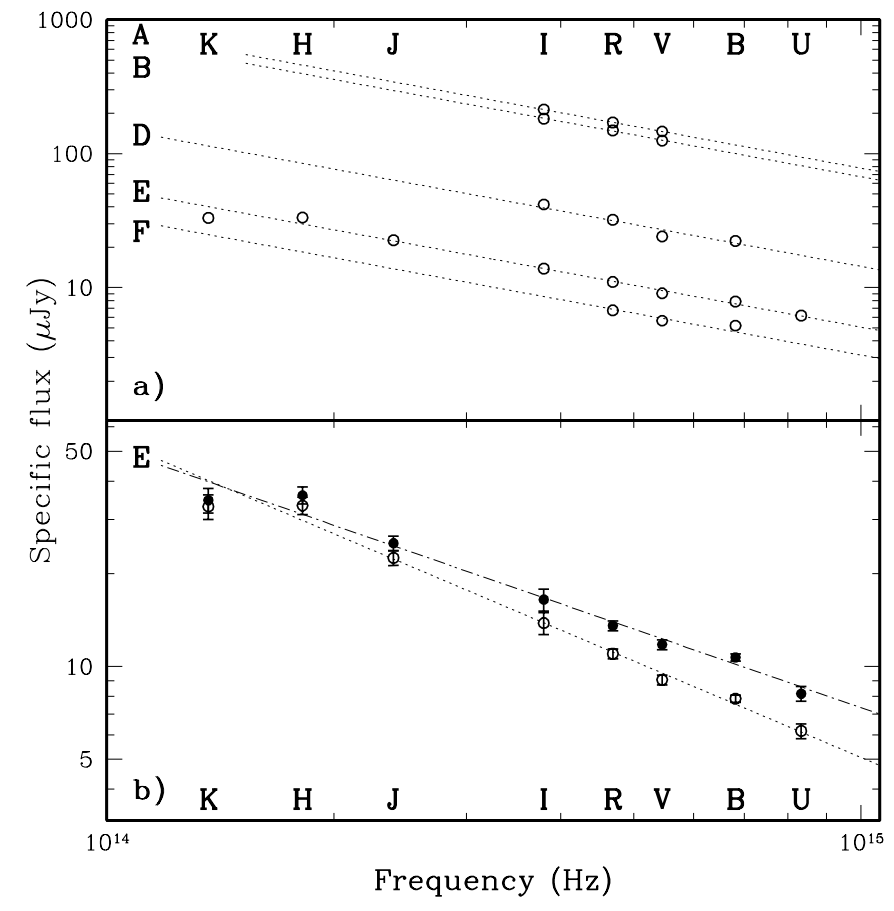

Fig. 3. a) Photometric spectra of GRB 028013 afterglow at various times (see Table 2), together with the best fit (dotted lines). Data are corrected for reddening in the Galaxy but not in the host. Error bars are smaller than the symbols and are not plotted. b) TNG spectrum on Aug. 15.04 UT; open points and dotted line are corrected only for Galactic reddening, while filled points and dot-dashed line are corrected also for reddening in the host (see text).

Table 2. Time evolution of the spectral index $\left(F_{v} \propto v^{-\alpha}\right)$. Galactic (but not intrinsic) extinction has been accounted for by assuming $A_{V}=0.42$.

\begin{tabular}{lllll}
\hline \hline UT & $\#$ & Telescope & Bands & $\alpha$ \\
\hline 13.28 & A & Baade $6.5 \mathrm{~m}$ & VRI & $1.06 \pm 0.06$ \\
13.31 & B & Baade $6.5 \mathrm{~m}$ & $V R I$ & $1.00 \pm 0.05$ \\
13.88 & C & AZT $1.1 \mathrm{~m}$ & RJK & $0.85 \pm 0.14$ \\
14.04 & D & Baade $6.5 \mathrm{~m}$ & BVRI & $1.15 \pm 0.05$ \\
15.04 & E & TNG $3.5 \mathrm{~m}$ & UBVRIJHK & $1.01 \pm 0.04$ \\
16.01 & F & TNG $3.5 \mathrm{~m}$ & BVR & $0.72 \pm 0.22$ \\
\hline All & - & - & - & $1.04 \pm 0.03$ \\
\hline
\end{tabular}

situation is GRB 010921, whose light curve might have displayed two breaks, the first only constrained by an early LOTIS upper limit to be at less than $\sim 1$ day (Park et al. 2002), and the second determined by means of HST observation after $\sim 35$ days (Price et al. 2003).

There are various interpretations to explain the presence of an achromatic break. If the blastwave producing the GRB is collimated, the break is expected to occur when the inverse of the bulk Lorentz factor of the ejecta $\Gamma$ equals the half-opening angle of the jet $\vartheta_{\text {jet }}$ (Rhoads 1999). In this context, Frail et al. (2001) found that the net energy released by GRBs, after correcting for beaming, is approximately constant for a number of events, to within a factor of $\sim 2$. This result is based on the observed correlation between break times and measured isotropic 


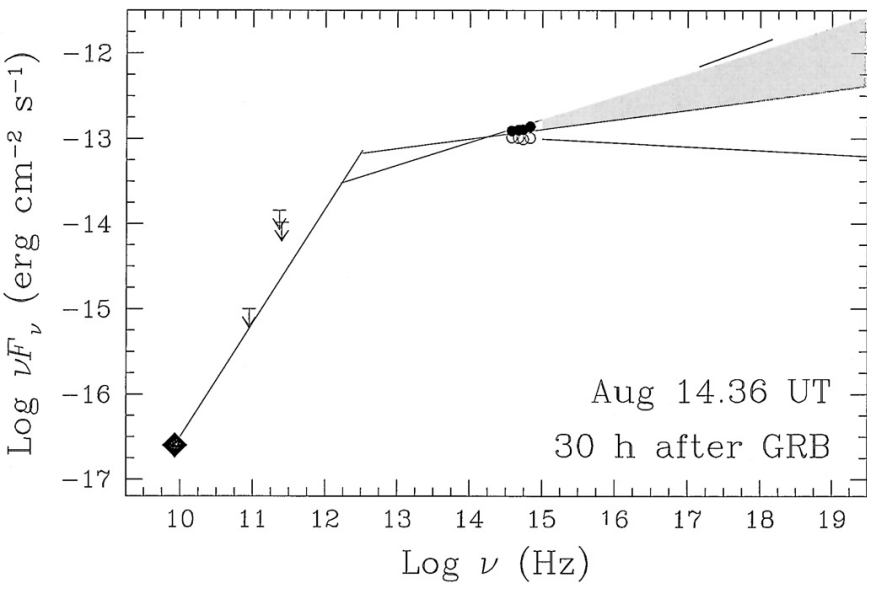

Fig. 4. Radio to X-ray spectral energy distribution of the afterglow of GRB 020813, $30 \mathrm{~h}$ after the trigger. Optical (BVRI bands) and X-ray data have been computed at the epoch of the radio detection by interpolating between different spectra and using the appropriate decay law. Open symbols are corrected only for Galactic extinction, while the additional host component has been taken into account for filled symbols. Radio detection together with the millimeter upper bounds limit the spectral slope $\left(F_{v} \propto v^{-\alpha}\right)$ to be $\alpha \geq-1 / 3$ at $\mathrm{GHz}$ frequencies (in particular a self-absorbed spectrum is ruled out). In the region between the millimeter and optical bands, the drawing is only indicative.

energies. Using the fluence $S=3.8 \times 10^{-5} \mathrm{erg} \mathrm{cm}^{-2}$ reported by Hurley et al. (2002) in the $(25 \div 100) \mathrm{keV}$ band, and applying a bolometric correction of $5.5 \pm 3.5$ (calculated in the same way as in Bloom et al. 2001), we get an isotropic energy ${ }^{5}$ of $(9.8 \pm 6.2) \times 10^{53} \mathrm{erg}$, ranking third in the sample of bursts with known redshift after GRB 990123 and GRB 000131. Using the formalism described by Frail et al. (2001), the opening angle of the jet is $\vartheta_{\text {jet }}=\left(1.7^{\circ} \pm 0.2^{\circ}\right) n_{-1}^{1 / 8} \eta_{20}^{1 / 8}$, where $n_{-1}$ is the external number density in units of $10^{-1} \mathrm{~cm}^{-3}$ and $\eta=20 \% \eta_{20}$ is the gamma-ray production efficiency. This is the smallest value ever reported for a GRB. The beaming-corrected energy is hence $(5.4 \pm 2.5) \times 10^{50} n_{-1}^{1 / 4} \eta_{20}^{1 / 4} \mathrm{erg}$, close to the value found by Frail et al. (2001) in their sample.

In a different interpretation, an early break can be caused by the onset of the nonrelativistic phase, when $\Gamma$ drops to unity (e.g. Dai \& Lu 1999). This interpretation has been proposed for GRB 010222, which shows spectral and temporal properties similar to the case presented here (Masetti et al. 2001). In order to stop the fireball so quickly, however, an extremely high external density $n \sim 10^{8} \mathrm{~cm}^{-3}$ is required (Panaitescu $\&$ Kumar 2000). Since the radius of the fireball at $t=t_{\mathrm{b}}$ is $R \sim 10^{16} \mathrm{~cm}$, the surrounding material must in this case have a hydrogen column density of $N_{\mathrm{H}} \sim n R \sim 10^{24} \mathrm{~cm}^{-2}$, and hence it must be completely ionized in order not to conflict with the $\mathrm{X}$-ray observation, which does not show a high value of $N_{\mathrm{H}}$ at the redshift of the host (Butler et al. 2003). These parameters are typical of a supernova remnant (e.g. Vietri \& Stella 1998). If this interpretation is correct, however, the radio spectrum is expected to be heavily self-absorbed; this is not the case, as can be seen in Fig. 4.

${ }^{5}$ We adopt a standard cosmology: $h_{0}=0.65, \Omega_{\mathrm{m}}=0.3, \Omega_{\Lambda}=0.7$.
To extract further information on the physical parameters of the explosion, we attempted to model the broad-band spectral energy distribution (SED) of the afterglow, including the radio detection (Frail \& Berger 2002), millimeter upper limits (Bremer \& Castro-Tirado 2002; Bertoldi et al. 2002), and X-ray data (Butler et al. 2003). The extrapolation of the optical spectrum lies well below the value observed in the X-ray band, which has moreover a harder spectrum (Fig. 4, dashed line). This could indicate that such emission constitutes a different component (due e.g. to the Compton process, such as in GRB 000926; Harrison et al. 2001). Alternatively, additional extinction could be responsible for the optical/X-ray mismatch; we therefore fitted again all optical/NIR datasets with a powerlaw allowing for additional absorption at $z=1.254$, adopting the extinction law of Cardelli et al. (1989). The best fit yields $\alpha=0.85 \pm 0.07$ with $A_{V}$ (host) $=0.12 \pm 0.04$, a small but non-negligible amount. We get $\chi^{2}=51$ for 14 degrees of freedom. The statistical improvement (with the respect to the case of no additional extinction) is admittedly low; however, in this case the optical spectrum becomes harder, and a single component can account for both the NIR/optical and X-ray emissions (see Fig. 4, shaded region). Moreover, the spectral indices in the two bands are strikingly similar: $\alpha_{\mathrm{X}}=0.85 \pm 0.04, \alpha_{\text {opt }}=0.85 \pm 0.07$. Last, we note that (independently on any extinction) the decay slopes are very well constrained, and yet in full agreement: $\delta_{\mathrm{X}}=1.38 \pm 0.06$, $\delta_{\text {opt }}=1.44 \pm 0.04$. This seems to be a fine tuning if the two components have a different origin. Using Chandra data, Butler et al. (2003) have reported no excess column density with respect to the Galactic value. However, our $A_{V}$ corresponds to a modest $N_{\mathrm{H}}=1.95 \times 10^{20} \mathrm{~cm}^{-2}$ (assuming a Galactic gas to dust ratio), a very small column to detect at a redshift of $z=1.254$ (the observed value is reduced by a factor $\left.\sim(1+z)^{3} \sim 10\right)$. In the following, we assume that indeed NIR/optical and X-ray emissions constitute a single component.

Since $\alpha_{\mathrm{X}}<1$, the spectrum is hard, with its peak frequency (in $v F_{v}$ ) lying above the $\mathrm{X}$-ray band. If the emission we see is synchrotron by a powerlaw distribution of electrons $\left(N(\gamma) \propto \gamma^{-p}\right)$, there are two possibilities, depending on whether the emitting particles are cooling rapidly or not. In the first case, $p=2 \alpha=1.6 \pm 0.1$ ("flat" distribution), while in the second case $p=2 \alpha+1=2.6 \pm 0.1$ (a more conventional value). Theoretical models predict several relations between the temporal and spectral slopes, depending on a number of assumptions such as the external density profile, cooling regime, and dynamical conditions. Before the achromatic break, however, the fireball should always follow a relativistic spherically symmetric evolution, and the predictions are robust and easy to check (e.g. Panaitescu \& Kumar 2000; Dai \& Cheng 2001). The only delicate issue regards the flat distribution case (when $p<2$ ), where the evolution of the spectrum is sensitive to the high-energy cutoff of the electron distribution, the behaviour of which is not well understood; we adopt the prescription given e.g. by Moderski et al. (2000) and Dai \& Cheng (2001). Table 3 shows all possible cases, presenting different ambient media and spectral shapes. None of them can account for the low value $\delta_{1}=0.78 \pm 0.02$. To eliminate such mismatch, a possible explanation is to invoke a strong Compton cooling (either 
Table 3. Predicted slopes and comparison with our observed values $\delta_{1}=0.78 \pm 0.02$ and $\delta_{2}=1.44 \pm 0.04$ for different models (all in the framework of a spherical relativistic fireball). $v_{\mathrm{i}}$ and $v_{\mathrm{c}}$ are the standard injection and cooling frequencies (defined e.g. in Sari et al. 1998). $v_{\mathrm{o}}$ spans the whole observed band, from optical $\left(\sim 10^{14} \mathrm{~Hz}\right)$ to $\mathrm{X}$-ray $\left(\sim 10^{18} \mathrm{~Hz}\right)$.

\begin{tabular}{llllll}
\hline \hline \multirow{2}{*}{$\begin{array}{l}\text { Frequencies } \\
\text { order }\end{array}$} & Ambient & Electron & Predicted & \multicolumn{2}{c}{ Discrepance } \\
& density & index $p$ & slope $\delta$ & $\delta_{1}$ & $\delta_{2}$ \\
\hline$v_{\mathrm{c}}<v_{\mathrm{i}}<v_{\mathrm{o}}$ & uniform & $1.6 \pm 0.1$ & $0.92 \pm 0.02$ & $7.5 \sigma$ & $13 \sigma$ \\
& wind & $1.6 \pm 0.1$ & $0.95 \pm 0.01$ & $9.0 \sigma$ & $12 \sigma$ \\
$v_{\mathrm{i}}<v_{\mathrm{o}}<v_{\mathrm{c}}$ & uniform & $2.6 \pm 0.1$ & $1.2 \pm 0.1$ & $4.3 \sigma$ & $2.4 \sigma$ \\
& wind & $2.6 \pm 0.1$ & $1.7 \pm 0.1$ & $9.3 \sigma$ & $2.6 \sigma$ \\
$v_{\mathrm{i}}<v_{\mathrm{c}}<v_{\mathrm{o}}$ & uniform & $1.6 \pm 0.1$ & $0.92 \pm 0.02$ & $7.5 \sigma$ & $13 \sigma$ \\
& wind & $1.6 \pm 0.1$ & $0.95 \pm 0.01$ & $9.0 \sigma$ & $12 \sigma$ \\
\hline
\end{tabular}

on self-synchrotron or external photons), whose emission is confined at high energies $(>10 \mathrm{keV})$. In this case, the cooling frequency should decrease slower with time, or even increase, and so the decay would be flatter above this frequency. Alternatively, a refreshed shock model (Sari \& Mészáros 2000) could also alleviate the discrepance, as recently proposed by Björnsson et al. (2002) for GRB 010222.

Acknowledgements. We thank Ümit Kiziloğlu for communicating to us his refined $R$-band measurement, Jens Hjorth, Leslie Hunt and Sergio Campana for useful discussion. We also thank Scott Barthelmy for maintaining the GCN system. FT and DM acknowledge the Italian MIUR for financial support.

\section{References}

Barth, A. J., Sari, R., Cohen, M. H., et al. 2003, ApJ, 584, L47 Bertoldi, F., Frail, D. A., Weiss, A., et al. 2002, GCN Circ., 1497 Beuermann, K., Hessman, F. V., Reinsch, K., et al. 1999, A\&A, 352, L26

Björnsson, G., Hjorth, J., Pedersen, K., \& Fynbo, J. U. 2002, ApJ, 579, L79

Bloom, J. S., Frail, D. A., \& Sari, R. 2001, AJ, 121, 2879

Bloom, J. S., Fox, D. W., \& Hunt, M. P. 2002, GCN Circ., 1476
Bremer, M., \& Castro-Tirado, A. J. 2002, GCN Circ., 1487

Butler, N. R., Marshall, H. L., Ricker, G. R., et al. 2003, ApJ, submitted [astro-ph/0303539]

Cardelli, J. A., Clayton, G. C., \& Mathis, J. S. 1989, ApJ, 345, 245

Costa, E., Frontera, F., Heise, J., et al. 1997, Nature, 387, 783

Covino, S., Malesani, D., Ghisellini, G., et al. 2002, GCN Circ., 1498

Dai, Z. G., \& Lu, T. 1999, ApJ, 519, L155

Dai, Z. G., \& Cheng, K. S. 2001, ApJ, 558, L109

Devillard, N. 1997, The Mess, 87

Fox, D. W., Blake, C., \& Price, P. A. 2002, GCN Circ., 1470

Frail, D. A., \& Berger, E. 2002, GCN Circ., 1490

Frail, D. A., Kulkarni, S. R., Sari, R., et al. 2001, ApJ, 562, L55

Gladders, M., \& Hall, P. 2002a, GCN Circ., 1472

Gladders, M., \& Hall, P. 2002b, GCN Circ., 1513

Gladders, M., \& Hall, P. 2002c, GCN Circ., 1514

Harrison, F., Bloom, J. S., Frail, D. A., et al. 1999, ApJ, 523, L121

Harrison, F., Yost, S. A., Sari, R., et al. 2001, ApJ, 559, 123

Hurley, K., Cline, T., Mazets, E., et al. 2002, GCN Circ., 1483

Israel, G. L., Marconi, G., Covino, S., et al. 1999, A\&A, 348, L5

Masetti, N., Palazzi, E., Pian, E., et al. 2001, A\&A, 374, 382

Mészáros, P. 2002, ARA\&A, 40, 137

Metzger, M. R., Djorgovski, S. G., Kulkarni, S. R., et al. 1997, Nature, 387,878

Moderski, R., Sikora, M., \& Bulik, T. 2002, ApJ, 529, 151

Panaitescu, A., \& Kumar, P. 2000, ApJ, 543, 66

Park, H. S., Williams, G. G., Hartmann, D. H., et al. 2002, ApJ, 571, L131

Piran, T. 1999, Phys. Rep., 314, 575

Predehl, P., \& Schmitt, J. H. M. M. 1995, A\&A, 293, 889

Price, P. A., Kulkarni, S. R., Schmidt, B. P., et al. 2003, ApJ, 584, 931

Rhoads, J. E. 1999, ApJ, 525, 737

Sagar, R., Stalin, C. S., Bhattacharya, D., et al. 2001, BASI, 29, 91

Sari, R., \& Mészáros, P. 2000, ApJ, 535, L33

Sari, R., Piran, T., \& Narayan, R. 1998, ApJ, 497, L17

Šimon, V., Hudec, R., Pizzichini, G., \& Masetti, N. 2001, A\&A, 377, 450

Stanek, K. Z., Garnavich, P. M., Kaluzny, J., Pych, W., \& Thompson, I. 1999, ApJ, 522, L39

Stanek, K. Z., Garnavich, P. M., Jha, S., et al. 2001, 563, 592

van Paradijs, J., Groot, P. J., Galama, T. J., et al. 1997, Nature, 386, 686

Vietri, M., \& Stella, L. 1998, ApJ, 507, L45

Villasenor, J., Graziani, C., Shirasaki, Y., et al. 2002, GCN Circ., 1471 